\title{
A Fast GEM Algorithm for Bayesian Wavelet-Based Image Restoration Using a Class of Heavy-Tailed Priors ${ }^{\star}$
}

\author{
José M. Bioucas-Dias \\ Instituto de Telecomunicações, \\ Instituto Superior Técnico, \\ Torre Norte, Piso 10, \\ Av. Rovisco Pais, \\ 1049-001 Lisboa, PORTUGAL \\ Phone: 351218418466 Email: bioucas@lx.it.pt
}

\begin{abstract}
The paper introduces modelling and optimization contributions on a class of Bayesian wavelet-based image deconvolution problems. Main assumptions of this class are: 1) space-invariant blur and additive white Gaussian noise; 2) prior given by a linear (finite of infinite) decomposition of Gaussian densities. Many heavy-tailed priors on wavelet coefficients of natural images admit this decomposition. To compute the maximum a posteriori (MAP) estimate, we propose a generalized expectation maximization (GEM) algorithm where the missing variables are the Gaussian modes. The maximization step of the EM algorithm is approximated by a stationary second order iterative method. The result is a GEM algorithm of $O(N \log N)$ computational complexity. In comparison with state-of-the-art methods, the proposed algorithm either outperforms or equals them, with low computational complexity.
\end{abstract}

\section{Introduction}

Image deconvolution is a longstanding linear inverse problem with applications in remote sensing, medical imaging, astronomy, seismology, and, more generally, in image restoration [1].

The challenge in many linear inverse problems is that they are ill-posed, i.e., either the linear operator does not admit inverse or it is near singular yielding

\footnotetext{
* This work was supported by the Fundação para a Ciência e Tecnologia, under the project POSI/34071/CPS/2000.
} 
highly noise sensitive solutions. To cope with the ill-posed nature of these problems, a large number of traditional techniques has been developed, most of them under the regularization or the Bayesian frameworks [2], [3],[4], [5], [6].

The heart of the regularization and Bayesian approaches is the a priori knowledge expressed by the prior/regularization term. A "good" prior should express knowledge about images being described. For example, the weak membrane [7], in the regularization setup, and the compound Gauss Markov random field [8], in the Bayesian setup were conceived to model piecewise-smooth images. This was an improvement over the classical quadratic priors.

Wavelet-based approaches have recently been adopted to solve linear inverse problems [9], [10], [11], [12], [13], [14], [15], [16]. Underlying this direction is the parsimonious representation provided by the wavelet transform of a large class of natural images [17]: images are essentially described by a few large wavelet coefficients. This fact has fostered Bayesian and regularization approaches where the prior favors a few large wavelet coefficients and many nearly zero ones (the so-called heavy-tailed priors).

In formulating linear space-invariant inverse problems in the wavelet domain, one is frequently faced with linear operations resulting from the composition of Toeplitz operators with the wavelet transforms. This composed operator is not diagonal and introduces unbearable computational complexity in the waveletbased deconvolution schemes. Recent works [18] and [16] have circumventing this difficulty by recognizing that each of these operations per se can be computed efficiently with fast algorithms.

\subsection{Proposed approach}

We introduce a wavelet-based Bayesian solution to image deconvolution. The observation mechanism comprehends space-invariant blur and additive Gaussian noise. The wavelet coefficients are assumed to be independent with density given by a linear (finite of infinite) combination of Gaussian densities. This class of densities models many heavy-tailed priors, namely, the Gaussian mixture models (GMM), the Jeffreys' non-informative prior [19], the Laplacian prior, the equivalent garrote prior (see [20] and papers therein).

To compute the MAP estimate, we propose an EM algorithm where the missing variables are the Gaussian modes. The maximization step of the EM algorithm includes a huge non-diagonal linear system with unbearable computational complexity. To avoid this difficulty we approximate the linear system solution by a few iterations of a stationary second order iterative method. The resulting 
scheme is a generalized expectation maximization (GEM) algorithm, achieving convergence in a few tens of iterations. The fast Fourier transform (FFT) and the discrete wavelet transform (DWT) are the heaviest computations on each GEM step. Thus the overall algorithm complexity is $O(N \log N)$.

In a set of experiments, the proposed algorithm either equals or outperforms state-of-the-art methods [10], [15], [16], [21], [12].

The paper is organized as follows. Section 2 formulates the restoration problem in the wavelet domain under the Bayesian framework. Section 3 introduces a class of heavy-tailed priors that can be expressed as linear combination of Gaussian terms. It is shown that this class contains many of the heavy-tailed priors used in wavelet-based image denoising and restoration. Still in Section 3 , it is introduced a generalized expectation maximization algorithm aimed at the fast computation of the maximum a posteriori image estimate. Finally, Section 4 presents experimental results illustrating the effectiveness of the proposed methodology.

\section{Problem formulation}

Let us denote $\mathbf{x}$ and $\mathbf{y}$ as vectors containing the true and the observed image gray levels, respectively, arranged in column lexicographic ordering. We assume, without loss of generality, that images are square of size $N$ (number of pixels).

The observation model herein considered is

$$
\mathbf{y}=\mathbf{H x}+\mathbf{n},
$$

where $\mathbf{H}$ is a square block-Toeplitz matrix accounting for space-invariant blur and $\mathbf{n}$ is a sample of zero-mean white Gaussian noise vector with density $p(\mathbf{n})=$ $\mathcal{N}\left(\mathbf{n} \mid \mathbf{0}, \sigma^{2} \mathbf{I}\right)[\mathcal{N}(\mathbf{z} \mid \mathbf{m}, \mathbf{C})$ denotes the Gaussian multivariate density of mean $\mathbf{m}$ and covariance $\mathbf{C}$ evaluated at $\mathbf{z}$, and $\mathbf{I}$ is the identity matrix].

Let $\mathbf{W}$ denote the orthogonal discrete wavelet transform (DWT) and $\boldsymbol{\theta}=$ $\mathbf{W} \mathbf{x}$ the wavelet coefficients of $\mathbf{x}$. Since $\mathbf{W}$ is orthogonal, expression (1) can be written as

$$
\mathbf{y}=\mathbf{H} \mathbf{W}^{T} \boldsymbol{\theta}+\mathbf{n} .
$$

The density of the observed vector $\mathbf{y}$ given $\boldsymbol{\theta}$ is then $p(\mathbf{y} \mid \boldsymbol{\theta})=\mathcal{N}\left(\mathbf{y} \mid \mathbf{H W}^{T} \boldsymbol{\theta}, \sigma^{2} \mathbf{I}\right)$. Given a prior $p(\boldsymbol{\theta})$, the maximum a posteriori (MAP) estimate of $\boldsymbol{\theta}$ is given by

$$
\begin{aligned}
\widehat{\boldsymbol{\theta}} & =\arg \max _{\boldsymbol{\theta}}\{\log p(\mathbf{y} \mid \boldsymbol{\theta})+\log p(\boldsymbol{\theta})\} \\
& =\arg \max _{\boldsymbol{\theta}}\left\{\frac{-\left\|\mathbf{y}-\mathbf{H} \mathbf{W}^{T} \boldsymbol{\theta}\right\|^{2}}{2 \sigma^{2}}+\log p(\boldsymbol{\theta})\right\} .
\end{aligned}
$$


As in many recent works, we assume that the wavelet coefficients are mutually independent and identically distributed, i.e.,

$$
p(\boldsymbol{\theta})=\prod_{i=1}^{N} p\left(\theta_{i}\right) .
$$

The independence assumption is motivated by the high degree of decorrelation exhibited by wavelet coefficients of natural images. Although decorrelation does not imply independence, the former has led to very good results.

If $\mathbf{H}=\mathbf{I}$, i.e., there is no blur, the image restoration at hand fall into a denoising problem. In this case the maximization (4) reduces to $N$ decoupled coefficient-wise maximizations, what can be efficiently solved exploiting the orthogonality of $\mathbf{W}$ and using fast implementations of the DWT (see, e.g. [20], $[22])$.

If $\mathbf{H} \neq \mathbf{I}$, i.e., there exists blur, the maximization (4) cannot be decoupled. Furthermore, matrix $\mathbf{H W}^{T}$ of size $N \times N$ introduces complexity beyond reasonable. In the next section we develop a GEM algorithm that avoids direct manipulation of matrix $\mathbf{H W}^{T}$.

\section{A GEM algorithm that avoids direct manipulation of $\mathrm{HW}^{T}$}

Let us assume that the prior on each wavelet coefficient is given by

$$
p(\theta)=E_{z}[p(\theta \mid z)],
$$

where $z$ is a continuous or discrete random variable, and

$$
p(\theta \mid z)=\mathcal{N}\left[\theta \mid 0, \sigma^{2}(z)\right] .
$$

Many of the heavy-tailed priors used in wavelet-based image denosing/restoration admit the decomposition implicit in the right-hand side of (5). Some examples are listed below (see [19])

- Gaussian mixture models (GMM): $z \in\{1, \ldots n\}$ and $P(z=i)$ is the probability of $\theta \sim \mathcal{N}\left[\theta \mid 0, \sigma^{2}(i)\right]$

- Laplacian prior: $p(z)=\gamma \exp (-\gamma z)$, with $z>0$, and $\sigma^{2}(z)=z$

- Jeffreys prior: $p(z) \propto 1 / z$, with $z>0$, and $\sigma^{2}(z)=z$.

- Any even prior such that $p(\sqrt{\theta})$ is completely monotone (see [23]). 
Random vectors $\mathbf{z} \equiv\left(z_{1}, \ldots, z_{N}\right)$ and $(\mathbf{y}, \mathbf{z})$ play the role of missing data and complete data, respectively, in our GEM formulation. The EM algorithm generates a nondecreasing sequence $[24]\left\{p\left(\mathbf{y}, \widehat{\boldsymbol{\theta}}_{t}\right), \mid t=0,1, \ldots\right\}$, where $\left\{\widehat{\boldsymbol{\theta}}_{t}, \mid t=\right.$ $0,1, \ldots\}$ is generated by the two-step iteration

\section{E-step:}

$$
\begin{aligned}
Q\left(\boldsymbol{\theta}, \widehat{\boldsymbol{\theta}}_{t}\right) & =E\left[\log \left[p(\mathbf{y}, \mathbf{z}, \boldsymbol{\theta}) \mid \mathbf{y}, \widehat{\boldsymbol{\theta}}_{t}\right]\right. \\
& =\frac{-\left\|\mathbf{y}-\mathbf{H} \mathbf{W}^{T} \boldsymbol{\theta}\right\|^{2}}{2 \sigma^{2}}-\frac{1}{2} \boldsymbol{\theta}^{T} \mathbf{D}_{t} \boldsymbol{\theta}+c^{t e}
\end{aligned}
$$

where $\mathbf{D}_{t} \equiv \operatorname{diag}\left\{E\left[\left(\sigma^{-2}\left(z_{1}\right), \ldots, \sigma^{-2}\left(z_{N}\right)\right) \mid \widehat{\boldsymbol{\theta}}_{t}\right]\right\}$ and $c^{t e}$ stands for constant. M-step:

$$
\begin{aligned}
& \widehat{\boldsymbol{\theta}}_{t+1}=\arg \max _{\boldsymbol{\theta}} Q\left(\boldsymbol{\theta}, \widehat{\boldsymbol{\theta}}_{t}\right) \\
& =\left(\sigma^{2} \mathbf{D}_{t}+\mathbf{W} \mathbf{H}^{T} \mathbf{H} \mathbf{W}^{T}\right)^{-1} \mathbf{W H}^{T} \mathbf{y} .
\end{aligned}
$$

M-step (9) is impracticable from the computational point of view, as it amounts to solving the linear system $\mathbf{A}_{t} \boldsymbol{\theta}=\mathbf{y}^{\prime}$, where $\mathbf{A}_{t} \equiv \sigma^{2} \mathbf{D}_{t}+\mathbf{W} \mathbf{H}^{T} \mathbf{H} \mathbf{W}^{T}$ and $\mathbf{y}^{\prime}=\mathbf{W} \mathbf{H}^{T} \mathbf{y}$, of size $N^{2}$ and involving the matrix $\mathbf{H W}^{T}$. We tackle this difficulty by replacing the maximization (9) with a few steps of an iterative procedure that increments $Q\left(\boldsymbol{\theta}, \widehat{\boldsymbol{\theta}}_{t}\right)$, with respect to $\boldsymbol{\theta}$. The resulting scheme is thus a GEM algorithm.

Let $\mathbf{A}_{t}=\mathbf{C}_{t}-\mathbf{R}$ be a splitting [25] of $\mathbf{A}_{t}$, where $\mathbf{C}_{t} \equiv\left(\sigma^{2} \mathbf{D}_{t}+\mathbf{I}\right)$ and $\mathbf{R} \equiv\left(\mathbf{I}-\mathbf{W} \mathbf{H}^{T} \mathbf{H} \mathbf{W}^{T}\right)$. Assuming that $\mathbf{A}_{t}$ is positive definite, then the secondorder iterative method defined by

$$
\begin{array}{rlr}
\mathbf{r}_{i} & =\mathbf{A}_{t} \boldsymbol{\xi}_{i}-\mathbf{y}^{\prime} & i=0,1, \ldots \\
\boldsymbol{\xi}_{1} & =\boldsymbol{\xi}_{0}-\beta_{0} \mathbf{C}_{t}^{-1} \mathbf{r}_{0} & \\
\boldsymbol{\xi}_{i+1} & =\alpha \boldsymbol{\xi}_{i}+(1-\alpha) \boldsymbol{\xi}_{i-1}-\beta \mathbf{C}_{t}^{-1} \mathbf{r}_{i} & i=1,2, \ldots,
\end{array}
$$

converges to the solution of $\mathbf{A} \boldsymbol{\theta}=\mathbf{y}^{\prime}$, if and only if

$$
\left\{\begin{array}{l}
0<\alpha<2 \\
0<\beta<2 \alpha / \lambda_{N}
\end{array}\right.
$$

where $0<\lambda_{1} \leq \lambda_{2} \leq \cdots \leq \lambda_{N}$ are the eigenvalues of $\mathbf{C}_{t}^{-1} \mathbf{A}_{t}$ (see Theorem 5.9 of $[25$, ch. 5$]$ ). The optimal convergence factor is

$$
\rho_{o p t} \equiv\left[1-\sqrt{\lambda_{1} / \lambda_{N}}\right]\left[1+\sqrt{\lambda_{1} / \lambda_{N}}\right]
$$


and is achieved for

$$
\left\{\begin{aligned}
\alpha & =\rho_{o p t}^{2}+1 \\
\beta & =2 \alpha /\left(\lambda_{1}+\lambda_{N}\right) \\
\beta_{0} & =\beta / \alpha .
\end{aligned}\right.
$$

Some algebra applied to the third line of (10) leads to

$$
\begin{aligned}
\boldsymbol{\xi}_{i+1}= & (\alpha-\beta) \boldsymbol{\xi}_{i}+(1-\alpha) \boldsymbol{\xi}_{i-1} \\
& +\beta \mathbf{C}_{t}^{-1}\left\{\boldsymbol{\xi}_{i}+\mathbf{W H}^{T}\left(\mathbf{y}-\mathbf{H} \mathbf{W}^{T} \boldsymbol{\xi}_{i}\right)\right\} \quad i=1,2, \ldots
\end{aligned}
$$

Expression shown in the second line of (10) is also given by (12) with $\alpha=1$ and $\beta=\beta_{0}$. Given that $\mathbf{C}_{t}=\left(\sigma^{2} \mathbf{D}_{t}+\mathbf{I}\right)$ is diagonal, the product $\mathbf{W} \mathbf{H}^{T} \mathbf{H} \mathbf{W}^{T} \boldsymbol{\xi}_{i}$, necessary to determine the residual $\mathbf{r}_{i}$, is the heaviest computation in each iteration (12). We note however that $\mathbf{W} \mathbf{H}^{T} \mathbf{H} \mathbf{W}^{T} \boldsymbol{\xi}_{i}$ can be computed efficiently, since there exists fast implementations $[O(N)]$ of the DWT and of the inverse DWT [17], and the product of a Toeplitz matrix by a vector can also be computed efficiently, by embedding $\mathbf{H}$ in a larger block-circulant matrix. Block-circulant matrices are diagonalized by the $2 \mathrm{D}$ discrete Fourier transform. Therefore, by using the $2 \mathrm{D}$ fast Fourier transform, the complexity of the product of a Toeplitz matrix by a vector is $[O(N \log N)][1]$.

A pertinent question is the choice of the number of iterations, say $p$, of the optimization step ${ }^{1}$ (O-step) assuring that $Q$ increases, i.e., $Q\left(\boldsymbol{\xi}_{p}, \widehat{\boldsymbol{\theta}}_{t}\right)>Q\left(\widehat{\boldsymbol{\theta}}_{t}, \widehat{\boldsymbol{\theta}}_{t}\right)$. A very simple solution to this problem consists in computing $Q\left(\boldsymbol{\xi}_{i}, \widehat{\boldsymbol{\theta}}_{t}\right)$ after each iteration (12) and check if $Q\left(\boldsymbol{\xi}_{i}, \widehat{\boldsymbol{\theta}}_{t}\right)>Q\left(\widehat{\boldsymbol{\theta}}_{t}, \widehat{\boldsymbol{\theta}}_{t}\right)$. Note that this procedure adds only a small computational complexity to the GEM algorithm, since the heaviest step in determining the quadratic function $Q\left(\xi_{i}, \widehat{\boldsymbol{\theta}}_{t}\right)$ given by $(7)$ is the computation of $\mathbf{H W}^{T} \boldsymbol{\theta}$, also needed to compute $\xi_{i}$ in (12).

Figure 1, part a), illustrates the behavior of $Q\left(\boldsymbol{\xi}_{i}, \widehat{\boldsymbol{\theta}}_{t}\right)$ and $\log p\left(\widehat{\boldsymbol{\theta}}_{t} \mid \mathbf{y}\right)$ for an image of size $256 \times 256$, blur uniform of size $9 \times 9$, and blurred signal-to-noise ratio of $40 \mathrm{~dB}$. Notice that, for a given $\widehat{\boldsymbol{\theta}}_{t}$, the plotted values of $Q\left(\boldsymbol{\xi}_{i}, \widehat{\boldsymbol{\theta}}_{t}\right)$ are strictly increasing. Part b) plots $\log p\left(\widehat{\boldsymbol{\theta}}_{t} \mid \mathbf{y}\right)$ as function of the total number of iterations (12), parameterized by $p$ (number of iterations (12) per O-step). Curves for $p=5$ and for $p=11$ are very close, whereas curve for $p=2$ is well below the others. This pattern of behavior was systematically observed and seems to indicate that for $p \geq p_{\text {min }}$, the evolution of $\log p\left(\widehat{\boldsymbol{\theta}}_{t} \mid \mathbf{y}\right)$ depends mainly on the total number of iterations (12). A deep study of the balance between the number of inner and

\footnotetext{
${ }^{1}$ From now on we refer to O-step instead of M-step, because $Q\left(\boldsymbol{\theta}, \widehat{\boldsymbol{\theta}}_{t}\right)$ is not maximized with respect to $\boldsymbol{\theta}$, but only increased.
} 

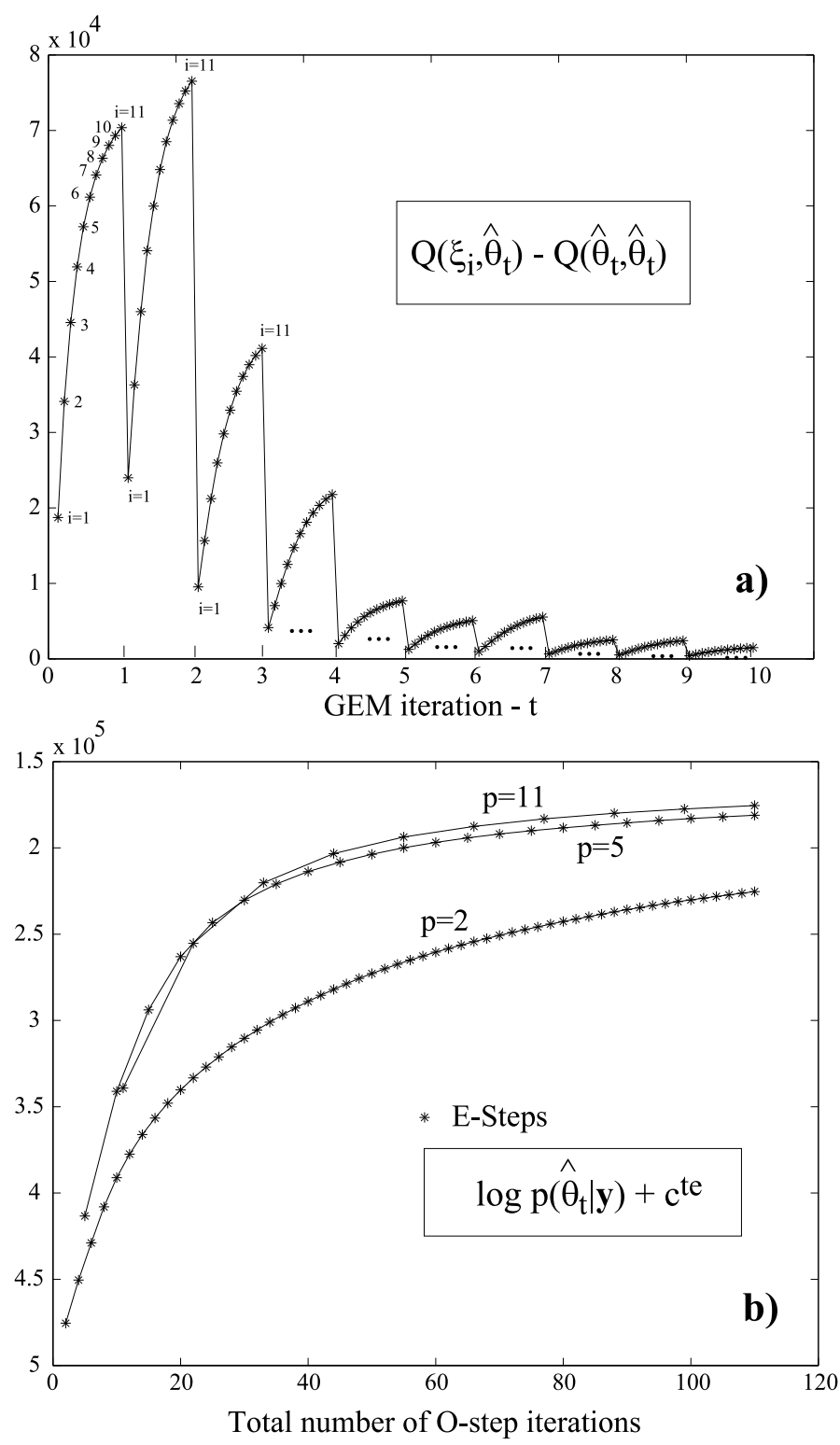

Fig. 1. a) Evolution of $Q\left(\boldsymbol{\xi}_{i}, \widehat{\boldsymbol{\theta}}_{t}\right)-Q\left(\widehat{\boldsymbol{\theta}}_{t}, \widehat{\boldsymbol{\theta}}_{t}\right)$, for $i=1,2, \ldots, 11$ and $t=0,1, \ldots, 10$; b) Evolution of $\log p\left(\widehat{\boldsymbol{\theta}}_{\boldsymbol{t}} \mid \mathbf{y}\right)$ (up to a constant $c^{t e}$ ) parameterized with $p$ (O-step iterations). 


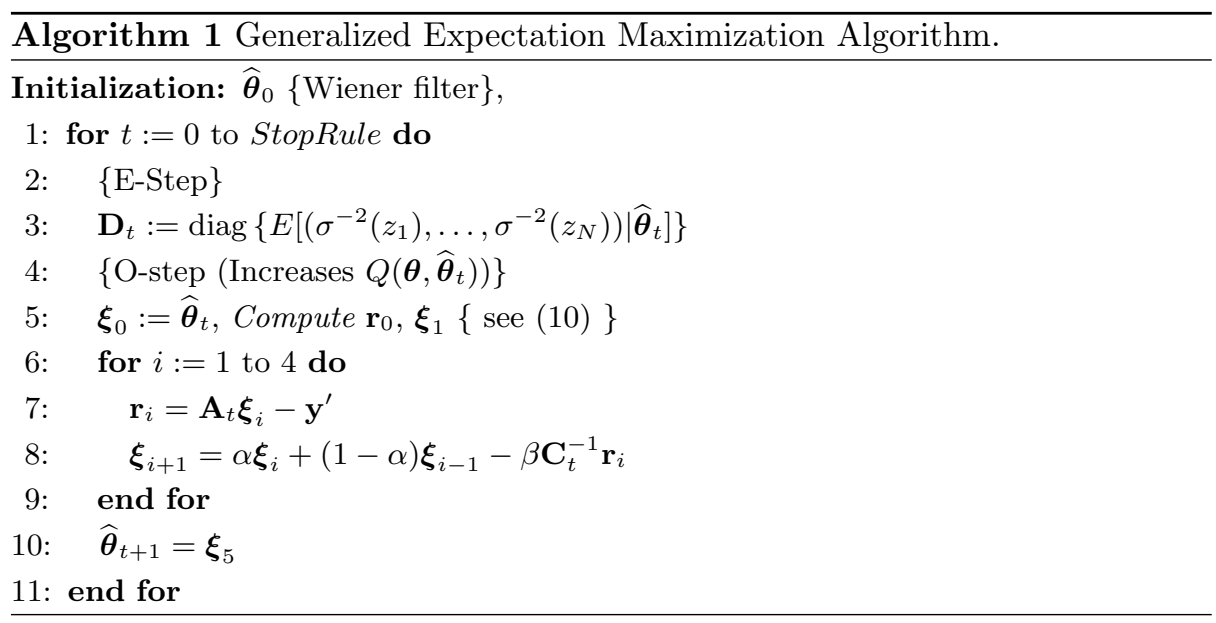

outer iterations is beyond the scope of this paper and should be addressed in future work.

Algorithm 1 shows the pseudo-code for the proposed GEM scheme. The number of iterations of the O-step is set to 4 . Given that $\mathbf{C}_{t}^{-1} \mathbf{A}_{t}=\mathbf{C}_{t}^{-1} \sigma^{2} \mathbf{D}_{t}+$ $\mathbf{C}_{t}^{-1} \mathbf{W} \mathbf{H}^{T} \mathbf{H} \mathbf{W}^{T}$ is positive definite, $0 \leq \lambda_{i}\left(\mathbf{C}_{t}^{-1} \sigma^{2} \mathbf{D}_{t}\right) \leq 1$, for $i=1, \ldots, N$, and $\mathbf{C}_{t}, \mathbf{D}_{t}$, and $\mathbf{W} \mathbf{H}^{T} \mathbf{H} \mathbf{W}^{T}$ are symmetric matrices, we have $0<\lambda_{i}\left(\mathbf{C}_{t}^{-1} \mathbf{A}_{t}\right) \leq$ $1+\lambda_{i}\left(\mathbf{C}_{t}^{-1} \mathbf{W} \mathbf{H}^{T} \mathbf{H} \mathbf{W}^{T}\right)$. Noting that $0<\lambda_{i}\left(\mathbf{C}_{t}^{-1}\right) \leq 1$ and that matrix $\mathbf{W}$ is unitary, it follows that $0<\lambda_{i}\left(\mathbf{C}_{t}^{-1} \mathbf{A}_{t}\right) \leq 1+\lambda_{N}\left(\mathbf{H H}^{T}\right)$. The approximation $\widetilde{\lambda}_{1}=0.01$ and $\widetilde{\lambda}_{N}=1+\lambda_{N}\left(\mathbf{H}^{T} \mathbf{H}\right)$ was taken, for $\lambda_{1}\left(\mathbf{C}_{t}^{-1} A_{t}\right)$ and $\lambda_{N}\left(\mathbf{C}_{t}^{-1} A_{t}\right)$, respectively. It should be stressed that, although this approximation might be rough, it assures that inequalities (11) are satisfied and is good enough to boost the converge rate by an order of magnitude when comparing with the first order iterative method obtained by setting $\alpha=1$ in (10) (see [25, ch. 5 ])).

We call attention for the following aspects of Algorithm 1:

- Unknown parameters: If there are unknown parameters in the observation model (e.g., observation noise $\sigma^{2}$ ) or in the prior, they can be inferred iteratively in the O-step.

- Computation of $\mathbf{D}_{t}$ : Matrix $\mathbf{D}_{t}$ depends on the type of prior. Below, we list a generic diagonal element $d_{t}=E\left[\sigma^{2}(z) \mid \theta_{t}\right]$ of $\mathbf{D}_{t}$ for four priors (see 
[19], [23], [20]):

$$
\begin{aligned}
\text { Gaussian mixture } d_{t} & =\frac{\sum_{i=1}^{n} \frac{P(z=i)}{\sigma_{i}^{2}} p\left(\theta_{t} \mid z=i\right)}{p\left(\theta_{t}\right)} \\
\text { Laplacian prior } d_{t} & =2 \gamma\left|\theta_{t}\right|^{-1} \\
\text { Jeffreys prior } d_{t} & =\left|\theta_{t}\right|^{-2} \\
\text { Garrote prior } d_{t} & =\frac{-\left|\theta_{t}\right|+\sqrt{\theta_{t}^{2}+4 a \sigma^{2}}}{2\left|\theta_{t}\right| \sigma^{2}}
\end{aligned}
$$

The denoising algorithm introduced in [20] is equivalent to the Garrote prior with $a=3$. The present formulation opens the door to adapting parameter $a$ to data.

- Translation-Invariant restoration: Translation- invariant (TI) waveletbased methods outperform the orthogonal DWT based ones, as the former significantly reduce the blocky artifacts associated to the dyadic shifts inherent to the orthogonal DWT basis functions [26]. In the present setup, replacing the orthogonal DWT with the TI-DWT does not alter the GEM nature of the developed algorithm, as the optimization step still increment the objective function $Q\left(\boldsymbol{\theta}, \widehat{\boldsymbol{\theta}}_{t}\right)$.

\section{Experimental results}

We now present a set of four experiments illustrating the performance of Algorithm 1. Original images are cameraman (experiments 1, 2, and 3,) and lena (experiment 4 ) both of size $256 \times 256$. Estimation results are compared with state-of-the-art methods [10], [15], [16], [21], [12]. In all experiments, we employ TI-DWT, with Haar wavelets (Daubechies-2), and the equivalent Garrote prior with $a=3$ as it yields the best results among priors compared in paper [16]. Noise is assumed unknown and the stopping rule is

$$
\frac{\left\|\widehat{\mathbf{x}}_{t+1}-\widehat{\mathbf{x}}_{t}\right\|_{2}}{\left\|\widehat{\mathbf{x}}_{t}\right\|_{2}}<2 \times 10^{-3} \sigma^{2} .
$$

In the first experiment we take the setup of [16]: blur uniform of size $9 \times 9$ and signal-to-noise-ratio of the blurred image (BSNR) set to BSNR $=40 \mathrm{~dB}$. In the second and third experiments we consider the setup of [16], [15]: point spread function of blur $h_{i j}=\left(1+i^{2}+j^{2}\right)$, for $i, j=-7, \ldots, 7$, and noise variances set to $\sigma^{2}=2$ for experiment 2 and $\sigma^{2}=8$ for experiment 3. Finally, in experiment 4 , we use the setup of [12]: $5 \times 5$ separarable blur filter with weights $[1,4,6,4,1] / 16$ and noise of standard variance $\sigma=7$. 
Table 1. SNR improvements (ISNR) of the proposed algorithm (Algoritm 1) and methods [10], [15], [16], [21], [12].

\begin{tabular}{c|cccc}
\hline & \multicolumn{5}{|c}{ ISNR (dB) } \\
Method & Exp1 & Exp2 & Exp3 & Exp4 \\
\hline Algoritm 1 & 8.10 & 7.47 & 5.17 & 2.73 \\
Figueiredo \& Nowak [16] & 7.02 & 7.22 & 5.06 & 2.42 \\
Neelamani et al. [21] & 7.30 & - & - & - \\
Banham \& Katsaggelos [10] & 6.70 & - & - & - \\
Jalobeanu et al. [15] & - & 6.75 & 4.85 & - \\
Liu \& Moulin [12] & - & - & - & 1.08 \\
\hline
\end{tabular}

Table 1 shows the signal-to-noise improvements (ISNR) of the proposed approach and methods [10], [15], [16], [21], [12], for the four experiments. Algorithm 1 outperforms the others in all experiments. The number of GEM iterations to satisfy the stop criterion was $55,10,8$, and 3 , for the experiments $1,2,3$, and 4 , respectively.

Figure 2a) shows the cameraman image, part b) is a degraded version (blur $(9 \times 9)$ uniform, $\mathrm{BSNR}=40 \mathrm{~dB})$, and part $\mathrm{c})$ is the restored image with Algorithm 1 , corresponding to a ISNR of 8.1dB.

Figure 3a) shows the cameraman image, part b) is a degraded version $\left(h_{i j}=\right.$ $\left(1+i^{2}+j^{2}\right)$, for $i, j=-7, \ldots, 7$, and noise variance set to $\left.\sigma^{2}=8\right)$, and part c) is the restored image with Algorithm 1, corresponding to a ISNR of $5.17 \mathrm{~dB}$.

Figure 4a) shows the lena image, part b) is a degraded version (blur with weights $[1,4,6,4,1] / 16$ and noise of standard variance $\sigma=7)$, and part c) is the restored image with Algorithm 1, corresponding to a ISNR of 2.73dB.

\section{Concluding remarks}

We developed a new fast Bayesian wavelet-based algorithm to image deconvolution. To compute the MAP estimate, we adopted a GEM optimization algorithm that employs a second order stationary iterative procedure to approximate the M-step of the EM algorithm. The total complexity is $O(N \log N)(N$ is the number of image pixels). In a set of experiments the proposed methodology competes with state-of-the-art methods. 

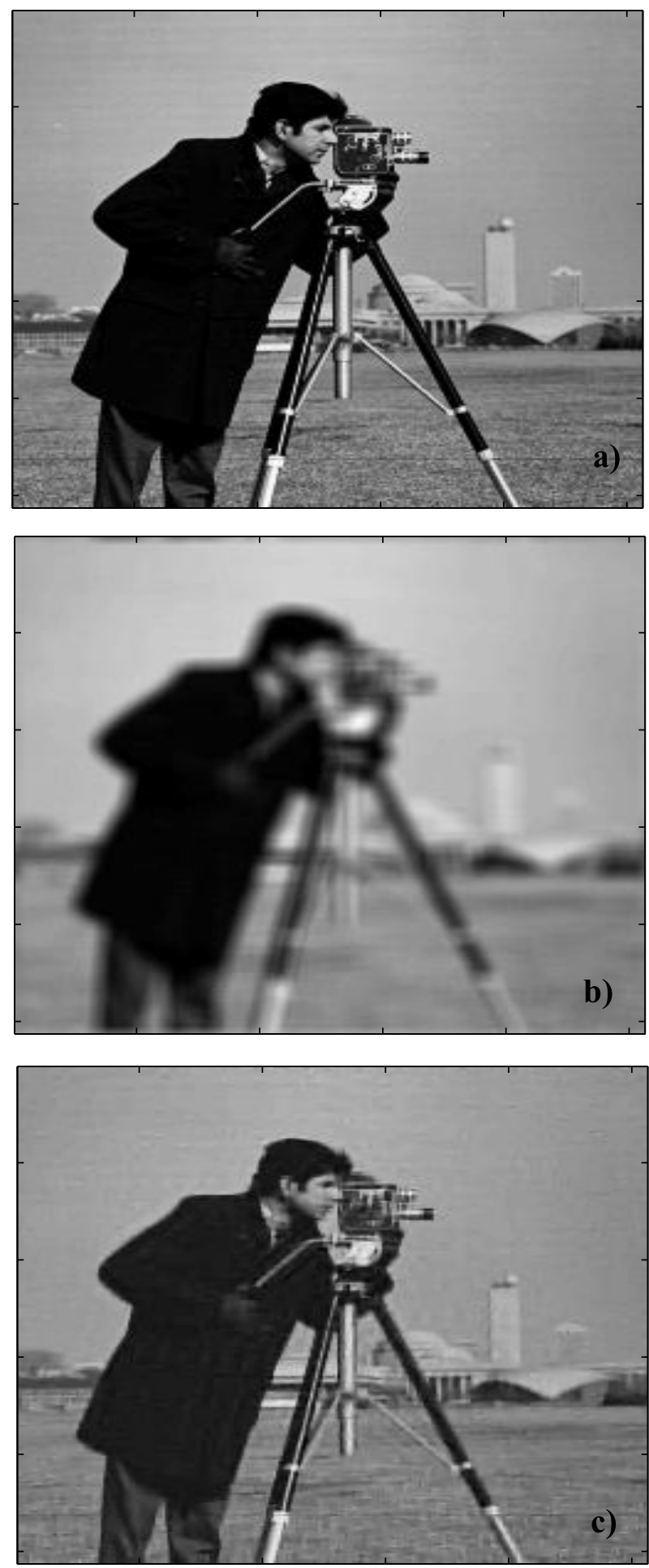

Fig. 2. Camera-man: a) Original image; b) Blurred noisy image (blur $(9 \times 9)$ uniform, $\mathrm{BSNR}=40 \mathrm{~dB}) ; \mathrm{c})$ Restored image with Algorithm 1 (ISNR $=8.1 \mathrm{~dB})$. 

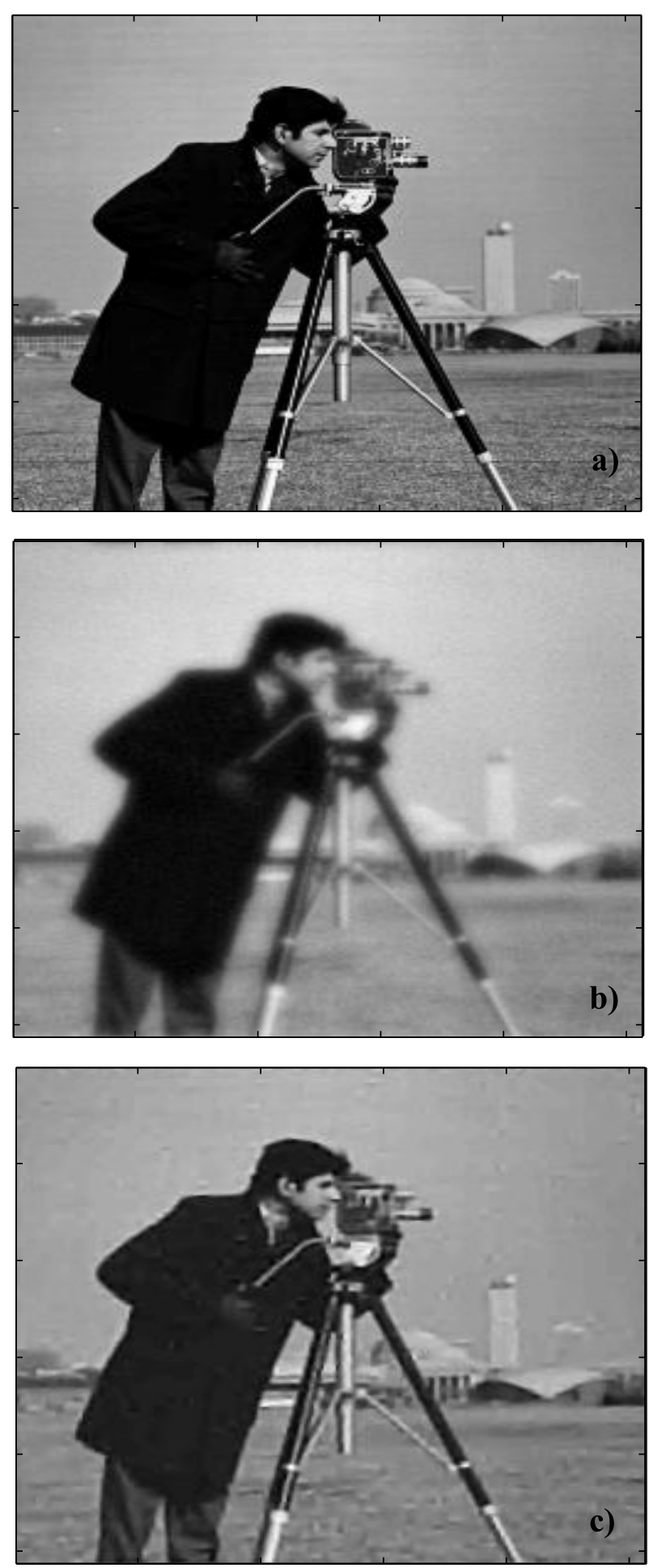

Fig. 3. a) Original image; b) Blurred noisy image (blur $h_{i j}=\left(1+i^{2}+j^{2}\right)$, for $i, j=$ $-7, \ldots, 7$, and noise variance set to $\sigma^{2}=8$ ); c) Restored image with Algorithm 1 (ISNR $=5.17 \mathrm{~dB}$ ). 

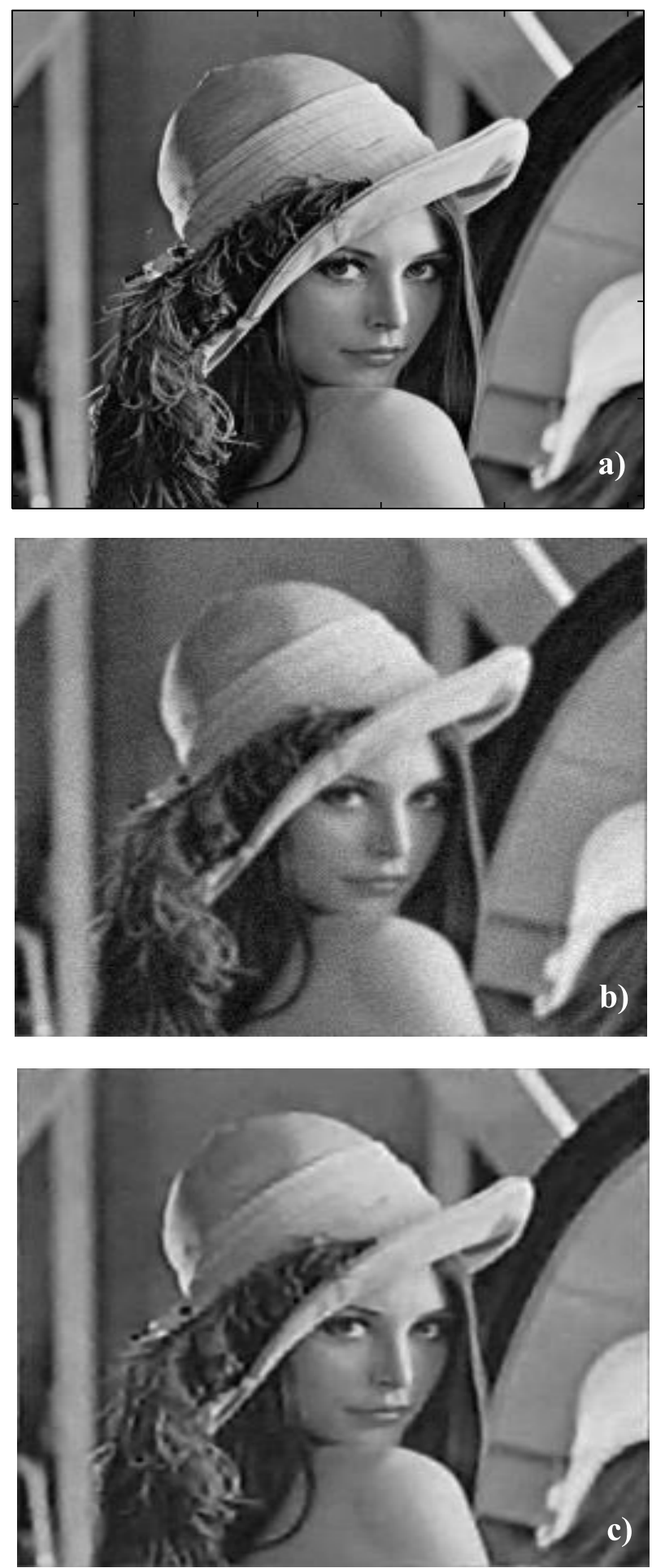

Fig. 4. a) Original image; b) Blurred noisy image (separable blur filter ( blur with with weights $[1,4,6,4,1] / 16$ and noise of standard variance $\sigma=7)$; b) Restored imaged Algorithm $1($ ISNR $=2.73 \mathrm{~dB})$. 


\section{References}

1. A. Jain. Fundamentals of Digital Image Processing. Prentice Hall, Englewood Cliffs, 1989.

2. S. Geman and D. Geman. Stochastic relaxation, Gibbs distribution and the Bayesian restoration of images. IEEE Transactions on Pattern Analysis and Machine Intelligence, PAMI-6(6):721-741, November 1984.

3. T. Poggio, V. Torre, and C. Koch. Computational vision and regularization theory. Nature, vol. 317:314-319, 1985.

4. D. Terzopoulos. Regularization of inverse visual problems involving discontinuities. IEEE Transactions on Pattern Analysis and Machine Intelligence, PAMI-8(4):413424, July 1986 .

5. A. Katsaggelos, editor. Digital Image Restoration. Spriger-Verlag, New York, 1991.

6. A. Katsaggelos, J. Biemond, R. Schafer, and R. Mersereau. A regularized iterative image restoration algorithm. IEEE Transactions on Signal Processing, 39(4):914929, April 1991.

7. A. Blake and A. Zisserman. Visual Reconstruction. MIT Press, Cambridge, M.A., 1987.

8. F. Jeng and J. Woods. Compound Gauss-Markov random fields for image processing. In A.Katsaggelos, editor, Digital Image Restoration, pages 89-108. Springer Verlag, 1991.

9. D. Donoho. Nonlinear solution of linear inverse problems by wavelet-vaguelette decompositions. Journal of Applied and Computational Harmonic Analysis, 1:100$115,1995$.

10. M. Banham and A. Katsaggelos. Spatially adaptive wavelet-based multiscale image restoration. IEEE Transactions on Image Processing, 5:619-634, 1996.

11. F. Abramovich, T. Sapatinas, and B. Silverman. Wavelet thresholding via a Bayesian approach. Journal of the Royal Statistical Society (B), 60, 1998.

12. J. Liu and P. Moulin. Complexity-regularized image restoration. Proc. IEEE Int. Conf. on Image Proc, pages 555-559, 1998.

13. Y. Wan and R. Nowak. A wavelet-based approach to joint image restoration and edge detection. In SPIE Conference on Wavelet Applications in Signal and Image Processing VII, Denver, CO, 1999. SPIE Vol. 3813.

14. J. Kalifa and S. Mallat. Minimax restoration and deconvolution. In P. Muller and B. Vidakovic, editors, Bayesian Inference in Wavelet Based Models. SpringerVerlag, New York, 1999.

15. A. Jalobeanu, N. Kingsbury, and J. Zerubia. Image deconvolution using hidden Markov tree modeling of complex wavelet packets. In Proceedings of the IEEE International Conference on Image Processing - ICIP'2001, Thessaloniki, Greece, 2001.

16. M. Figueiredo and R. Nowak. An em algorithm for wavelet-based image restoration. IEEE Transactions on Image Processing, 2003. Accepted for publication (available in htt://www.lx.it.pt/ mtf/). 
17. S. Mallat. A Wavelet Tour of Signal Processing. Academic Press, San Diego, 1998.

18. R. Neelamani, H. Choi, and R. Baraniuk. Wavelet-based deconvolution using optimally inversion for ill-conditioned systems. In Wavelet Applications in Signal and Image Processing, volume 3169, pages 389-399, Oct. 2001.

19. C. Robert. The Bayesian Choice. A Decision-Theoritic Motivation. SpringerVerlag, 1994.

20. M. Figueiredo and R. Nowak. Wavelet-based image estimation: an empirical Bayes approach using Jeffreys' noninformative prior. IEEE Transactions on Image Processing, 10(9):1322-1331, 2001.

21. R. Neelamani, H. Choi, and R. Baraniuk. Wavelet-based deconvolution for illconditioned systems. IEEE Transactions on Image Processing, 2001 (submitted).

22. P. Moulin and J. Liu. Analysis of multiresolution image denoising schemes using generalized - Gaussian and complexity priors. IEEE Transactions on Information Theory, 45:909-919, 1999.

23. F. Girosi. Models of noise and robust estimates. Massachusetts Institute of Technology. Artificial Intelligence Laboratory (Memo 1287) and Center for Biological and Computational Learning (Paper 66), 1991.

24. A. Dempster, N. Laird, and D. Rubin. Maximum likelihood estimation from incomplete data via the EM algorithm. Journal of the Royal Statistical Society B, 39:1-38, 1977.

25. O. Axelsson. Iterative Solution Methods. Cambridge University Press, New York, 1996.

26. R. Coifman and D. Donoho. Translation invariant de-noising. In Wavelets and Statistics, Lecture Notes in Statistics, pages 125-150, New York, 1995. SpringerVerlag. 